\title{
Cognitive Manipulation of Pain ${ }^{1}$
}

\author{
Richard E. Nisbett² and Stanley Schachter \\ Columbia University
}

\begin{abstract}
The experiment tests the notion that naturally occurring states of physiological arousal are manipulable in the same way that drug-induced arousal states have proven to be. The state of arousal studied is that produced by pain from electric shock. All subjects were given a placebo before the shock aperience and half were told that the side effects would cause arousal simptoms such as palpitation, tremor, cte. The other half expected no such symptoms. Subjects believing themselves to be in an artificial state of arousal failed to attribute their shock-created arousal to the shock, and found the shock less painful and were willing to tolerate inore of il. This "relabeling" of a naturally occurring state was shown to occur only for subjects in a relatively low state of fear.
\end{abstract}

In experiments conceived initially as studies of emotion, it has been repeatedly demonstrated that the "emotional labels" attached to aroused bodily states are, in good part, cognitively determined (Schachter, 1964; Schachter and Singer, 1962). These studies involved the manipulation of bodily state by the injection of epinephrine or placebo. In some conditions subjects were told the specific physiological symptoms, if any, to expect as a consequence of injection. In other conditions subjects did not expect that there would be such side effects. Where subjects experienced a state of arousal and were led to expect no such side effects, they proved readily manipulable into states of euphoria, anger, anxiety, and the like. Similar subjects, experimentally provided with an explanation for their aroused statc, were almost completely nommanipulab!c. This "plasticity" has been interpreted in terms of evaluative needs and labeling processes. Where an individual experiences a state of arousal for which he has no ready explanation, he labels this state in terms of cognitive and situational fartors. It has been further demonstrated (Jatané and Schachter,

${ }^{1}$ The research described in this palol was supported by grant $\mathrm{MH} 05203$ from the National Institute of Mental Health, United States Public Health Service, and by grant $G 23758$ from the National Science Foundation. The authors wish to express their warmest thanks to Harvey London for his assistance in the design and execution of the experiment.

'Presently at Yale University. 
1962; Schachter and Wheeler, 1962; Singer, 1963) that the intensity of the resulting emotion is a function of level of arousal.

These demonstrations of the plasticity of interpretation of bodily state have depended upon the experimental trick of simultaneously and independently manipulating physiological and cognitive factors. In nature, of course, cognitive or situational factors trigger physiological processes, and the triggering stimulus usually imposes the label we attach to our feelings. We see the threatening object; this perception-cognition initiates a state of sympathetic arousal, and this joint cognitive-physiological experience is labeled "fear."

Several considerations suggest that the line of reasoning guiding the experimental studies of emotion may be extended to these naturally occurring states and that the intensity of such states may be as modifiable as are expcrimentally induced states of arousal. As an example of this possibility, consider pain. Broadly, we can conceive of the intensity of experienced pain and of one's willingness to tolerate pain as a function of the intensity of stimulation of the pain receptors, of the autonomic correlates of such stimulation, and of a host of cognitive and situational factors. To the extent that we can convince a subject undergoing electric shock that his shock-produced symptoms and arousal state are due, not to shock, but to some outside agent such as a drug, he should, following the above considerations, experience less pain and be willing to tolerate more shock. Such an individual would, of course, regard his arousal as a drug-produced state rather than an indicator of pain or fear.

Since we would not expect that an individual undergoing extreme pain, fear, or rage could easily be persuaded to attribute the accompanying physiological arousal to an artificial suuree, there should be linuils placed on the generality of these notions. We should be able to alter the labeling of a bodily state only within a range bounded at the lower end by the cxistcnec of at least some arousal, and at the upper end by experiences so extreme that no amount of manipulation of cognitions will persuade the individual to attribute his bodily state to an artificial source. Common sense would indicate that no amount of argument would persuade a man dodging machine-gun bullets that his physiological arousal was due to anything but the exigencies of his situation. The present experiment examines the extent to which relabeling processes affect pain thresholds under conditions of high and low fear.

\section{PROCEDURE}

\section{Overview}

The experimental test of these ideas required (a) administration of a placebo, (b) the "natural" production of an aroused bodily state by a painful stimulus, 
(c) manipulation of the extent to which this arousal rould he attributed to the placebo, and (d) measurement of the extent to which the pain stimulus was tolerated and labeled as painful.

The experiment was described to subjects as one on skin sensitivity where the test of sensitivity would be electric shock. They were given a bricf lecture on the importance of shock sensitivity research for the prevention of accidents and the control of shock dosage for patients undergoing electroshock therapy. This was followed by a description of the shock stimulus which the subject was to take. The shock was described to one group as being extremely painful, and to the other as being mild and easily tolerable.

Subjects were then told that the experimenters were interested in the effects on skin sensitivity of a drug called Suproxin. The drug was described as being a mild one that could do no harm, but which had certain transitory side effects which would last for 15 or 20 minutes. The description of the "side effects" of the placebo differed across conditions. In one set of conditions, the "side effects" were actually symptoms produced by the shock. In the other pair of conditions, the symptoms were irrelevant to shock. After taking the placebo tablet, subjects were told that they could expect the side effects to start within 2-4 minutes. They were then left to wait alone for a 10-minute period.

After the waiting period, subjects were taken into a room containing the shock apparatus. Electrodes were attached and subjects were given a series of shocks of gradually increasing intensity. Subjects reported when they could first feel the shock, when the shock first became painful, and when the shock was too painful to endure.

\section{Manipulating Fear}

High fear. After describing the nature of the research, the experimenter concluded his patter with, "So what we'll ask you to do is very simple. We'd like to give you a series of electric shocks. Now I should be honest with you and tell you what you're in for. These shocks will hurt, they will be painful. If we're going to learn anything at all in this kind of research, it's necessary that the shocks be intense. What we'll do is hook some electrodes on your hand and give you a series of electric shocks. Again, I want to be honest with you and tell you that these shocks will be quite painful, but of course they won't do any permanent damage." The experimenter then continued with the instructions on Suproxin, the subjects took the pill and then waited alone for 10 minutes. At this point they were taken to the experimental room, the instructions about the painfulness of the shock repeated and the subjects given a sample "standardizing" shock which, to reinforce the manipulation, was a rather jarring and unpleasant 55 microamperes.

Low fear. In introducing the experiment, the experimenter reassured the subject by saying "Before you get worried, let me tell you not to let the word 'shock' bother you. There's not going to be any discomfort and I'm sure you'll enjoy the experiment." He then continued his description of the research, concluding with, "So what we will ask you to do will be very simple. We would like to give you is series of very mild electric shocks. What you will feel will be more like a tickle or a tingle than anything unpleasant. So we will put some electrodes on your hand ind give you a series of very mild shocks." The subjects then took the pill, waited 10 minutes, and wore given a sample shock of a harely perreptible 22 microamperex. 


\section{Manipulating Attribution of Bodily State}

The extent to which bodily state could be attributed to an external source was manipulated by creating a condition in which subjects expected, as "side effects" of the placebo, symptoms which would actually be caused by the shock (Pill Attrib condition). A condition was also created in which subjects expected symptoms which were irrelevant to the shock (Shock Attrib condition).

Pill Attrib subjects were told the following about the side effects of the placebo: "What will happen is that you may have some tremor, that is, your hand will start to shake, you will have some palpitation, that is, your heart will start to pound, and your rate of breathing may increase. Also, you will probably get a sinking feeling in the pit of your stomach, like butterflies." All of these are symptoms which were widely reported by pretest subjects who introspected about their physiological reactions to shock. To the extent that the manipulation is effective, subjects in this condition should atttribute these shock-produced symptoms to the pill.

Shock Attrib subjects were told: "What will probably happen is that your feet will feel numb, you may have an itching sensation over parts of your body, and you may get a slight headache." None of these symptoms, of course, is produced either by the shock or the placebo. It seemed a distinct possibility that reeling off a list of symptoms, any symptoms, might make a subject more introspective and concerned with his bodily state. Therefore we employed this technique of "false" symptoms, rather than telling a subject that the pill would have no effects at all, as a means of making the two attribution conditions somewhat more comparable. Subjects in this condition, then, will experience the physiological symptoms produced by shock and anticipation of shock and, since no plausible alternative exists, will perforce attribute these symptoms to the shock experience.

After the 10-minute waiting period, subjects were reminded of the symptoms appropriate to their condition, and told that they were probably just starting. A second reminder immediately preceded the administration of the shock series.

In summary, there were four conditions; Hi Fear Pill Attrib, Hi Fear Shock Attrib, Lo Fear Pill Attrib, and Lo Fear Shock Attrib. There were 16 subjects in each of the Hi Fear groups and 12 in each of the Lo Fear groups. All were male volunteers from introductory psychology courses at Long Island University.

\section{Measurement}

Pain thresholds. After the 10-minute waiting period, the subject was ushered into the experimental room and introduced to a second experimenter who proceeded to apply the electrodes to the fingers of the subject's left hand, to administer the sample "standardizing" shock, and to explain the procedure to the subject. It was explained that a series of shocks would be administered and that they would progressively increase in intensity. Shocks were administered every 15 seconds and the shock proper had a duration of .10 second. Starting with a subthreshold 20 microamperes, each successive shock in the early part of the series was roughly 5-10 microamperes more intense than the preceding shock. This gap widened during the series to an average of about 100 microamperes. These intervals were determined on the basis of pretests to be subjectively equal increments. There were a total of $\mathbf{3 7}$ steps in the shock series, with the final step delivering 3000 microamperes of current.

Subjects were requested to report (1) when they first folt the shock (sensitivity 
threshold), (2) when the shock first became painful (pain threshold), and (3) when the shock was too painful to endure and they wanted it stopped (tolerance threshold).

The subjects were told that when they reached a point too painful to endure, the shocks would be terminatcd. If a subject endured the entire 37 steps of the series without so complaining, the experiment automatically ended after the thirtyseventh trial.

The experimenter who administered the shocks was aware of the fear condition of the subject, but was totally ignorant of his attribution condition.

Questionnaires. In order to get measures of the effectiveness of the fear manipulation, subjects answered a questionnaire about "gencral physical and mental" state at the very end of the 10-minute waiting period. Embedded among a series of dummy items were two relevant scales dealing with how frightened they werc about taking shock and how worried they were about the effects of the shock.

At the cnd of the shock series, the subjects answered a questionnaire concerned with the painfulness of the shocks, the extent to which the subject experienced the symptoms which had been described, when they occurred, and the extent to which they were attributed to the placebo.

\section{A Note on the Noncomparability of Fear Conditions}

As must be evident from the introduction to this paper, the primary interests of this study are the examination of (1) the effects of the symptom-attribution manipulation on pain, and (2) the interaction of the attribution and fear manipulations. In no sense were we directly concerned in this experiment with the effects of manipulated fear on pain. Because of these interests we deliberately sacrificed the elegance of a completely symmetrical experimental design in order to maximize the effects of the fear manipulation. For example, in the Hi Fear condition the sample shock was jolting and painful, while in the Lo Fear condition it was simply a tickly sensation. The language used in describing the various thresholds was slightly different in high and low fear conditions, e.g., for the sensitivity threshold, Lo Fear subjects were asked to note when they "first felt a tingle," while Hi Fear subjects noted when they first felt a "shock." Without question experimental touches such as these made Hi Fear subjects more fearful and Lo Fear subjects less fearful than they might otherwise have been. It is the case, however, that such variations in procedure make direct comparison of $\mathrm{Hi}$ and Lo Fear subjects dubious. Among other things, for example, the sample shock may have provided a different point of reference for the two groups of subjects in making judgments of pain. Similarly, describing thresholds somewhat differently to $\mathrm{Hi}$ and Lo Fear subjects obviously will affect threshold values. For reasons such as these direct comparison of $\mathrm{Hi}$ and Lo Fear conditions is meaningless, and we shall not discuss the effects of the fear manipulation on the dependent variable.

Within fear conditions, of course, the pairs of experimental conditions are identical in cvery respect other than the attribution manipulation.

\section{RESULTS}

The experimental test of the hypotheses requires first, the successful manipulation of fear; second, the actual production of symptoms described in the Pill Attrib condition; and, finally, the successful manipulation of attribution. 
Effectiveness of fear manipulation. Evaluating first the effect of the fear manipulation, it can be seen in Table 1 that $S \mathrm{~s}$ in the Hi fear conditions report significantly more fear and worry after the waiting period than do subjects in the Lo Fear conditions. Means in Table 1 are for responses on four-point rating scales $(0=$ not at all, $3=$ extremely) to the questions "How frightened are you about taking shock?" and

TABLE 1

Mean Reponted fear and Worry Scores after Waiting Period

\begin{tabular}{ccc}
\hline Condition & Fear & Worry \\
\hline Lo Fear Pill Attrib. & .46 & .42 \\
Lo Fear Shock Attrib. & .58 & .31 \\
Hi Fear Pill Attrib. & 1.00 & .70 \\
Mi Fear Shock Attrib. & .92 & .911 \\
$t$ Hi vs. Lo & 2.50 & 2.78 \\
$p$ & $<.02$ & $<.01$ \\
\hline
\end{tabular}

"How worried are you about the effects of the shock?" The fear manipulation was clearly successful.

The level of physiological arousal. Table 2 presents the proportion of subjects in each condition who reported actually experiencing one or more of the symptoms (palpitation, tremor, breathing rate change, butterflies in the stomach) that we associate with receiving or anticipating shock. These data are simply tabulations of answers to the questions ahout each

TABLE 2

Proportion of Subjects Who Reported Arousal Symproms

$\begin{array}{lcc}\text { Condition } & N & \begin{array}{c}\text { \% Subjects reporting } \\ \text { 'true' shock-produced } \\ \text { symptoms }\end{array} \\ \text { Lo Fear Pill Attrib. } & 12 & 75.0 \\ \text { Lo Fear Shock Attrib. } & 12 & 83.3 \\ \text { Hi Fear Pill Attrib. } & 16 & 75.0 \\ \text { Ifi Fear Shock Altrib. } & 16 & 81.3 \\ \text { Total } & 56 & 78.6\end{array}$

of these symptoms on the questionnaire administered at the end of the shock portion of the experimental session. There are no significant differences between conditions, and obviously the large majority of subjects in each of the conditions report experiencing one or more of the physiological symptoms associated with shock.

That subjects actually did experience these symptoms and are not simply being suggestible or accommodating is indicated by a comparison 
of these figures with the proportion of subjects reporting the irrelcvant symptoms. While $78.6 \%$ of all subjects reported experiencing the true symptoms described in the Pill Attrib manipulation, only $21.2 \%$ of all subjects reported one or more of the "false" symptoms (itching skin, numb feet, headache) described in the Shock Attrib conditions.

Effectiveness of Attribution Manipulation. From their answers to the postexperimental questionnaire, most of the subjects who reported any arousal at all could be categorized as either attributing all of their symptoms to the pill or as attributing some or all of their symptoms to shock or fear. The frequencies in these categories are reported in Table 3. It can be seen that in Lo Fear conditions the manipulation has worked. Some $60 \%$ of the Pill Attrib subjects attribute their symptoms to the pill, while only one of eight Shock Attrib subjects do so. Where the shock is presumed to be mild and harmless, the subjects accept the immediately provided explanation and attribute the shock-induced symptoms to the pill rather than the shock. In marked contrast, the overwhelming majority of Hi Fear subjects, regardless of attribution condition, attribute their symptoms to the shock. This, of course, is precisely what was anticipated, for the Hi Fear conditions were deliberately made as frightening as possible in order to provide subjects with a plausible alternative explanation for their bodily state. Obviously the intensity of the Hi Fear manipulation has made it more plausible for subjects in these conditions to attribute their arousal to shock or fear than to the pill.

TABLE 3

Attribution of Symproms

\begin{tabular}{ccc} 
Condition & $\begin{array}{c}\text { Exclusive } \\
\text { attribution } \\
\text { to placebo }\end{array}$ & $\begin{array}{c}\text { Some attribution } \\
\text { to shock or fear }\end{array}$ \\
\hline Lo Fear Pill Attrib. & 6 & 4 \\
Lo Fear Shock Attrib. & 1 & 7 \\
Hi Fear Pill Attrib. & 2 & 10 \\
Hi Fear Shock Attrib. & 1 & 9 \\
\hline
\end{tabular}

\begin{tabular}{cl} 
Comparison & $p^{n}$ \\
\hline Lo PA vs. Hi PA & .05 \\
Lo PA vs. Hi SA & .03 \\
Lo PA vs. Lo SA & .05 \\
Lo PA vs. All Others & .01
\end{tabular}

Note. Entries are the number of subjects with the designated attribution. The total for each condition is less than the number of subjects in the condition because some subjects reported no symptoms or could not be categorized as to attribution.

${ }^{a} p$ values are Fisher's exact $p$. 
Clearly, the experimental conditions necessary to test the hypotheses have been established. The subjects actually experienced symptoms of physiological arousal, the manipulation of fear was successful, and the attribution of symptoms conforms to the manipulations in the Lo Fear and not in the Hi Fear condition.

Effect of the manipulation on tolerance of shock. Our formulation of the pain experience as, in part, a function of the intensity of arousal symptoms and of the attribution of these symptoms leads us to expect, first, that the attribution manipulation will have effects only in the Lo Fear conditions, and second, that these effects will be manifested only when arousal symptoms are prominent. In Lo Fear conditions, then, we should anticipate marked differences in Tolerance Threshold, for at this point of "unbearablensss" the arousal symptoms produced by shock and anticipation are undoubtedly at a maximum. In contrast, the attribution manipulation should have no effect on Sensitivity Threshold, for this is the point at which subjects first report that they are aware of some (nonpainful) sensation, and shock-produced arousal symptoms are nil. The effects on Pain Threshold should depend on the extent to which this "first sign of pain" is arousing. A low intensity pain is considerably less autonomically arousing (Valins, 1965) than is an intense pain. We should, then, expect the attribution manipulation to have relatively small effects on Pain Threshold. In Hi Fear, on the other hand, there should be no difference between the attribution conditions on any of the thresholds.

The extent to which the data support these expectations can be evaluated in Table 4, which presents the means for each of these thresholds. The figures in this table represent the average step in the series of 37 increasingly intense shocks at which subjects reported that they had reached a particular threshold. It can be seen that in the Lo Fear conditions, as anticipated, there is no difference in Scnsitivity Threshold, a small difference in Pain Threshold, and a marked and extremely large difference in Tolerance Threshold. Translating these steps into amperage, the average Pill Attribution subject was able to tolerate 1450 microamperes while the Shock Attribution subjects found 350 microamperes, on the average, intolerable. Obviously, the attribution of shock-produced symptoms to the pill has had a profound effect on the subject's ability or willingness to withstand pain.

In Hi Fear conditions pill and shock attribution subjects are similar on all three thresholds. As shown previously, the Hi Fear manipulation was successful enough to compel almost all of these subjects to attributc. their symptoms to being shocked, and they behaved accordingly.

Testing the Fear $\times$ Attribution interaction, on the Pain Threshold an 
TABLE 4

Mean Shock Thresholds

\begin{tabular}{cccc}
\hline Conditions & $\begin{array}{c}\text { Sensitivity } \\
\text { Threshold }\end{array}$ & $\begin{array}{c}\text { Pain } \\
\text { Threshold }\end{array}$ & $\begin{array}{c}\text { Tolerance } \\
\text { Threshold }\end{array}$ \\
\hline Lo Fear Pill Attrib. & 4.58 & 11.58 & 25.75 \\
Lo Fear Shock Attrib. & 4.58 & 8.00 & 15.75 \\
$t$ & 0 & 1.55 & 2.90 \\
$p$ & n.s. & $11 . s$. & $<.01$ \\
Hi Fear Pill Attrib. & 5.44 & 15.06 & 26.31 \\
Hi Fear Shock Attrib. & 5.13 & 19.31 & 28.19 \\
$t$ & .63 & 1.47 & 0.59 \\
$p$ & 11.5. & n.s. & n.s. \\
\hline
\end{tabular}

interaction $\ell$ test yields $\iota=-2.01$ wilh $p=.05$. For the Tolerance Threshold, the interaction $t=2.52$ with $p<.02$. The attribution manipulation affects the willingness to withstand pain only in the Lo Fear conditions.

In addition to these differences in tolerance of pain, there is evidence that the Lo Fear Pill Attrib subjects consciously experienced less pain than subjects in other conditions. On the post-experiment questionnaire, subjects were asked how painful they found the last shock they were willing to endure. The means for these responses are presented in Table 5 .

TABLE 5

Mean Reported Pain of Last Shock

\begin{tabular}{|c|c|c|}
\hline Condition & & Pain \\
\hline Lo Fear Pill Attrib. & & 1.37 \\
\hline Lo Fear Shock Attrib. & & 1.79 \\
\hline Hi Fear Pill Attrib. & & 2.16 \\
\hline Hi Fear Shock Attrib. & & 1.86 \\
\hline & $t$ & $p$ \\
\hline Lo PA vs. All others & 2.34 & $<.05$ \\
\hline
\end{tabular}

It can be seen that the Lo Fear Pill Attrib subjects reported less pain than any of the other groups, despite the fact that they actually tolerated as much shock as subjects in any other group. A comparison of the mean for the Lo Fear Pill Attrib group vs. all other groups yields a $t$ of 2.34 that is significant beyond the .05 level.

Finally, the categorization of subjects, in Table 3, into those who attributed their symptoms to the shock or fear vs. those who attributed their symptoms exclusively to the drug, suggests another means of analyzing the data. To the extent that subjects in any condition attributed 
their symptoms to the drug, they should have behaved like subjects in the Lo Fear Pill Attrib condition. If the tolerance threshold scores of the ten subjects in Table 2 who attributed their symptoms to the pill are contrasted with those of the thirty subjects who attributed their symptoms mainly to fear or the shock, it is found that four of the ten subjects in the former category were willing to take more shock than the shock apparatus could deliver, while this was true of none of the thirty subjects in the latter category. This difference is significant at the .003 level by Fisher's exact test. Further, the mean reported pain of the last shock for the pill-attributers is 1.53 and that for the shock-or-fear-attributers is 2.16. This difference yields a $t$ of 2.83 , which is significant beyond the .01 level.

The gist of the data is clear. Where subjects attribute shock-produced autonomic symptoms to the pill, they tolerate far more shock and report considerably less pain than when they attribute these symptoms to the shock proper. Earlier studies have demonstrated the cognitive manipulability of bodily states produced by the injection of epinephrine. The present study demonstrates that, within the limits of plausibility, the labeling of naturally occurring bodily states is similarly manipulable.

\section{REFERENCES}

Latané, B., ANd Schachier, S. Adrenalin and avoidance learning. Journal of Comparative and Physiological Psychology, 1962, 65, 369-372.

Schachrer, S. The interaction of cognitive and physiological determinants of emotional state. In P. H. Leiderman and D. Shapiro (Eds.), Prychobiological approaches to social behavior. Stanford, Calif.: Stanford Univer. Press, 1964. Pp. 138-173.

Schachter, S., and Singer, J. Cognitive, social and physiological determinants of emotional state. Psychological Review, 1962, 69, 379-399.

Schachter, S., ANd Wheeler, L. Epinephrine, chlorpromazine and amusement. Journal of Abnormal and Social Psychology, 1962, 65, 121-128.

Singer, J. E. Sympathetic activation, drugs and fright. Journal of Comparative and Physiological Psychology, 1963, 56, 612-615.

VALins, S. Emotionality and autonomic reactivity. Unpublished doctoral dissertation, Columbia University, 1964.

(Received November 1, 1965) 